\title{
Politiken der Naturgestaltung
}


Daniela Gottschlich · Tanja Mölders (Hrsg.)

\section{Politiken der \\ Naturgestaltung \\ Ländliche Entwicklung \\ und Agro-Gentechnik \\ zwischen Kritik und Vision}

Springer VS 
Herausgeberinnen

Daniela Gottschlich

Lüneburg, Deutschland
Tanja Mölders

Hannover, Deutschland

Das Projekt „PoNa - Politiken der Naturgestaltung. Ländliche Entwicklung und AgroGentechnik zwischen Kritik und Vision“" wurde vom Bundesministerium für Bildung und Forschung (BMBF) im Förderschwerpunkt Sozial-ökologische Forschung (SÖF) unter dem Kennzeichen 01UU0903 gefördert.
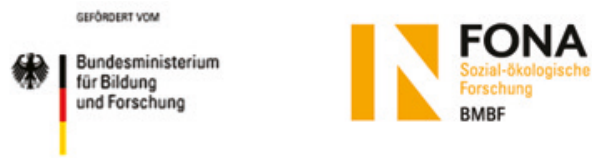

ISBN 978-3-658-08192-8

ISBN 978-3-658-08193-5 (eBook)

DOI 10.1007/978-3-658-08193-5

Die Deutsche Nationalbibliothek verzeichnet diese Publikation in der Deutschen Nationalbibliografie; detaillierte bibliografische Daten sind im Internet über http://dnb.d-nb.de abrufbar.

\section{Springer VS}

(C) Springer Fachmedien Wiesbaden GmbH 2017

Das Werk einschließlich aller seiner Teile ist urheberrechtlich geschützt. Jede Verwertung, die nicht ausdrücklich vom Urheberrechtsgesetz zugelassen ist, bedarf der vorherigen Zustimmung des Verlags. Das gilt insbesondere für Vervielfältigungen, Bearbeitungen, Übersetzungen, Mikroverfilmungen und die Einspeicherung und Verarbeitung in elektronischen Systemen.

Die Wiedergabe von Gebrauchsnamen, Handelsnamen, Warenbezeichnungen usw. in diesem Werk berechtigt auch ohne besondere Kennzeichnung nicht zu der Annahme, dass solche Namen im Sinne der Warenzeichen- und Markenschutz-Gesetzgebung als frei zu betrachten wären und daher von jedermann benutzt werden dürften.

Der Verlag, die Autoren und die Herausgeber gehen davon aus, dass die Angaben und Informationen in diesem Werk zum Zeitpunkt der Veröffentlichung vollständig und korrekt sind. Weder der Verlag noch die Autoren oder die Herausgeber übernehmen, ausdrücklich oder implizit, Gewähr für den Inhalt des Werkes, etwaige Fehler oder Äußerungen.

Gedruckt auf säurefreiem und chlorfrei gebleichtem Papier

Springer VS ist Teil von Springer Nature

Die eingetragene Gesellschaft ist Springer Fachmedien Wiesbaden GmbH

Die Anschrift der Gesellschaft ist: Abraham-Lincoln-Str. 46, 65189 Wiesbaden, Germany 


\section{Danksagung}

Dieser Band dokumentiert Ergebnisse des Projekts „PoNa - Politiken der Naturgestaltung" und damit Produkte und Prozesse einer fünfjährigen Forschungsarbeit. Ein solcher Band kann nie vollständig sein, kann nie alles und alle abbilden. Dass diese Publikation dennoch mehr ist als ein Sammelband und die Ergebnisse unserer Forschungsarbeit synthetisiert und integriert, verdanken wir dem engagierten Mitwirken einer Vielzahl von Menschen. Unser großer Dank gilt den wissenschaftlichen Mitarbeiter_innen Annemarie Burandt, Beate Friedrich, Jędrzej Sulmowski und Anna Szumelda. Sie haben sich mit uns auf „PoNa als inter- und transdisziplinäres Experiment in der Sozial-ökologischen Forschung" (wie es in Kapitel IV.2 dieses Bandes heißt) eingelassen und damit zugleich ihre eigene wissenschaftliche Qualifikation vollzogen. Ergänzt wurde dieses Team von weiteren wissenschaftlichen und studentischen Mitarbeiter_innen, die insbesondere die Endphase der vorliegenden Publikation mit Sorgfalt und Umsicht begleitet haben. Unser großer Dank gilt Lea Koch für ihre hervorragende Redaktionsarbeit, Michael Colshorn und Steffi Roth für die sorgfältige Pflege unserer Literaturdatenbank, Jana Koltzau für die Durchsicht des Literaturzeichnisses sowie Lars Johannsen für die Unterstützung bei EDVund Formatfragen. Boris Jarecki danken wir für die Unterstützung beim Korrekturlesen auf den letzten Metern.

Zum erweiterten PoNa-Team zählen wir auch all jene Begleiter_innen aus Wissenschaft und Praxis, die uns an unterschiedlichen Stationen unseres Forschungsprozesses wertvolle Impulse gegeben haben, die ihren Niederschlag auch in diesem Band finden. Stellvertretend nennen möchten wir an dieser Stelle unsere Mentorin Prof. Dr. Sabine Hofmeister sowie die zahlreichen Interviewpartner_innen, die ihr Wissen und ihre Zeit mit uns geteilt haben. Dankbar sind wir außerdem Prof. Dr. Matthias Bergmann und Dr. Engelbert Schramm, die mit uns über fünf Jahre hinweg Integrationsarbeit praktiziert haben und bereit waren, die Eindrücke und Einschätzungen dieser Zusammenarbeit in einem Beitrag zu reflektieren. Dr. Jan Kruse danken wir für drei anregende Methodenworkshops; sein früher Tod im Mai 2015 hat uns sehr getroffen.

Schließlich danken wir Dr. Petra Schilling für ihr sorgfältiges Lektorat und Sabine Schöller vom Springer VS Verlag für die gute Zusammenarbeit.

Nicht zuletzt gilt unser Dank dem Bundesministerium für Bildung und Forschung (BMBF), das uns finanziell gefördert und damit sehr gute Bedingungen für wissenschaftliche Qualifikation geschaffen hat. Diesen Dank adressieren wir vor allen an Claudia Müller, unsere Kontaktperson beim Projektträger Deutsches Zentrum für Luft- und Raumfahrt (DLR).

Wir wünschen allen Leser_innen eine anregende Lektüre!

Daniela Gottschlich \& Tanja Mölders als Herausgeberinnen 


\title{
Vorwort
}

\author{
Sabine Hofmeister
}

Der vorliegende Band bildet die Ergebnisse der Nachwuchsgruppe „PoNa Politiken der Naturgestaltung“ ab. Aufbauend auf der Analyse der wechselseitigen Beziehungen zwischen Natur und Politik werden hier entlang der Politikfelder Ländliche Entwicklung und Agro-Gentechnik die ökonomischen Rationalitäten und Mechanismen, die auf die Gestaltung gesellschaftlicher Naturverhältnisse einwirken, in kritischer Absicht aufgedeckt. Es wird gezeigt, dass und wie Widersprüche und Zielkonflikte in Bezug auf die Gestaltung gesellschaftlicher Naturverhältnisse - anstatt zum Gegenstand politischer Aushandlungen zu werden - unsichtbar gehalten werden. Das Forschungsprojekt PoNa will dabei nicht nur die Ursachen sozial-ökologischer Krisen (besser) verstehen, sondern zugleich auch Transformationswissen für eine nachhaltige Gestaltung gesellschaftlicher Naturverhältnisse erarbeiten. Dieses von sechs Nachwuchswissenschaftler_innen im Rahmen der sozial-ökologischen Nachhaltigkeitsforschung getragene Vorhaben ist nach meiner Einschätzung enorm mutig - mindestens aus drei Gründen:

In „PoNa - Politiken der Naturgestaltung“ werden Grenzen überschritten Grenzen zwischen Fachdisziplinen und Grenzen zwischen Wissenschaften und außerwissenschaftlicher Lebenswelt. Zugleich dringt die Forschung aber in den Kern der Wissenschaften vor - in jene Räume der akademischen Welt, in denen Menschen zu Wissenschaftler_innen, gemacht' werden, in denen sie sich wissenschaftlich qualifizieren: Das in PoNa generierte Wissen kondensiert in vier Promotions- und zwei Habilitationsprojekten. (Wie) Aber verträgt sich Grenzüberschreitung mit dem Erfordernis, sich innerhalb der Grenzen der Wissenschaft einzurichten?

In „PoNa - Politiken der Naturgestaltung“ werden Erkenntnisse kritischer Wissenschaften aufgenommen, Synergien - z. B. zwischen Nachhaltigkeits- und Geschlechterforschung - herausgearbeitet und theoriekreativ angewendet mit dem Ergebnis, Nachhaltigkeitswissenschaften als transformative, kritische Wissenschaft zu positionieren und die in der Sozial-ökologischen Forschung enthaltenen emanzipatorischen Potenziale freizulegen. (Wie) Aber verträgt sich die Kritik (auch) an Wissenschaft mit dem Schaffen von Wissen in der Wissenschaft? 
In „PoNa - Politiken der Naturgestaltung“ wird quer gedacht. Wissenschaftliche Verfahren werden quer zu den in den verschiedenen Fachdisziplinen etablierten Methodensets kombiniert und integriert, um Erkenntnisse zu generieren, die sich gerade nicht gradlinig in den Kanon wissenschaftlicher Forschungsergebnisse einreihen lassen. Eingebettet in einen sorgfältig und immer wieder (selbst)kritisch reflektierten Rahmen - entlang des normativen Leitziels einer nachhaltigen Entwicklung - wird das anscheinend Selbstverständliche gründlich infrage gestellt; es werden Forschungsfragen umformuliert und entlang von anders gestellten Fragen werden andere Erkenntnisse generiert. (Wie) Aber lassen sich lebensweltliche Problemlagen in der ,alten" Welt des Akademischen neu formulieren? (Wie) Lassen sich mit den ,alten' Begriffen und Instrumenten Wege zur Nachhaltigkeit entdecken, Problemlösungen ,neu' denken?

Tatsächlich ist es, schaut man in den vorliegenden Band hinein, der Forschungsnachwuchsgruppe PoNa gelungen, im ,alten Haus' der akademischen Welt neues Denken zu ermöglichen und neues Wissen zu schaffen. Dies mag nur Wenigen an nur wenigen Orten gelingen. Welche Orte braucht es also, um diese neue Art des Wissen-Schaffens erproben und entwickeln zu können? Welche Orte gibt es, an denen Neudenken als akademischer Erfolg gewertet und wertgeschätzt werden kann? Das Projekt „PoNa - Politiken der Naturgestaltung“ hat folgende institutionellen Experimentierräume für sich entdeckt und sich angeeignet:

Im Rahmen des Förderprogramms Sozial-ökologische Forschung des Bundesministeriums für Bildung und Forschung (BMBF) darf nicht nur, es soll auf neue Weise Neues gedacht werden: Nachwuchswissenschaftler_innen qualifizieren sich durch inter- und transdisziplinäre Forschung. An den Schnittstellen von Umwelt-, Natur- und Geisteswissenschaften sollen die Nachwuchsgruppen eigenverantwortlich selbst ausgewählte Forschungsaufgaben bearbeiten und dabei zugleich die Kultur interdisziplinären wissenschaftlichen Arbeitens pflegen und entwickeln. Außeruniversitäre und universitäre Forschung wirken ineinander, verbinden sich miteinander zu etwas Neuem. Einen solchen Förderrahmen braucht es, um Menschen zu ermutigen, neues Denken zu wagen und eine neue Form der Wissenschaft (Mode 2) weiterzuentwickeln.

Es braucht außerdem einen universitären Ort, an dem inter- und transdisziplinär erzeugtes Wissen, das sich zudem zu seinen normativen Grundlagen bekennt, das nicht nur transformativ, sondern auch emanzipativ wirken will, als wissenschaftliches Wissen Anerkennung findet. Die Fakultät Nachhaltigkeit an der Leuphana Universität Lüneburg ist dieses Wagnis eingegangen und hat die dafür notwendigen Rahmenbedingungen im Promotionskolleg Nachhaltigkeitswissenschaft geschaffen. An dieser Fakultät finden Nachwuchswissenschaftler_innen, die neues Wissen auf neue Weise schaffen wollen, einen Ort - einen 
Ort, an dem sie wertvolle wissenschaftliche Arbeit leisten und dafür wertgeschätzt werden.

Es braucht also geeignete Institutionen. Es braucht aber auch Menschen, die solche institutionellen Möglichkeitsräume zu nutzen verstehen, die sich einlassen auf die schwierige Gratwanderung zwischen ,neuen' und ,alten“ Wissenschaftswelten. In den Institutionen braucht es Forscher_innen, die eigenverantwortlich und eigensinnig, mutig und beharrlich, (selbst)kritisch und (selbst)bewusst sind, um sich jenen komplexen Fragen zu stellen, die im herkömmlichen Wissenschaftsbetrieb nicht einmal gestellt und schon gar nicht mit dem Ziel der akademischen Qualifikation bearbeitet werden können.

Die Sozial-ökologische Forschung, die Fakultät Nachhaltigkeit an der Leuphana Universität Lüneburg, Annemarie Burandt, Beate Friedrich, Daniela Gottschlich, Tanja Mölders, Jędrzej Sulmowski und Anna Szumelda haben sich von 2008 bis 2014 im Forschungsgebiet Umweltplanung am Institut für Nachhaltigkeitssteuerung zusammengefunden, um genau das jetzt geschafft zu haben! Der vorliegende Band gibt einen großartigen Einblick sowohl in das komplexe Forschungsfeld von PoNa als auch in die hierfür gewählte komplexe Art des Forschens.

Als Mentorin dieser außergewöhnlichen Forschungsnachwuchsgruppe habe ich - durch viel kreatives Chaos hindurch - sehr viel gelernt und ebenso viel Freude an dieser Zusammenarbeit erfahren dürfen. Ich wünsche den Leser_innen, dass sie diese Lernfreude mit mir teilen. 


\section{Inhaltsverzeichnis}

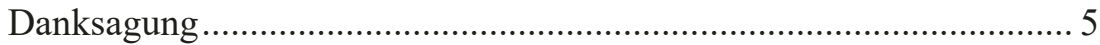

Daniela Gottschlich, Tanja Mölders

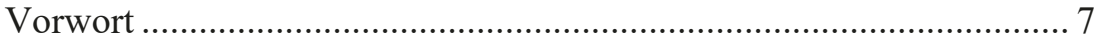

Sabine Hofmeister

Abbildungs- und Tabellenverzeichnis.................................................... 15

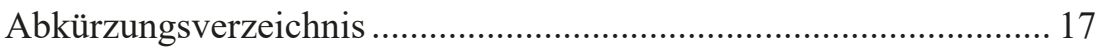

\section{TEIL I: Einleitung und theoretische Verortung}

1. Einleitung - Eine sozial-ökologische Forschungsperspektive ....21 Tanja Mölders, Daniela Gottschlich

2. Normative Orientierungen - Ein kritisch-emanzipatorisches Nachhaltigkeitsverständnis.......................................................... 31 Daniela Gottschlich, Tanja Mölders

3. Die paradoxen Hybride - Ein graphisches Essay über die Praxis der Unterscheidung zwischen Natur und Gesellschaft ..... 45 Jędrzej Sulmowski

\section{TEIL II: Ländliche Entwicklung als Politiken der Naturgestaltung}

1. Einführung in das Politikfeld Ländliche Entwicklung - Zwischen Wettbewerbsfähigkeit und Nachhaltigkeit 65 Tanja Mölders, Annemarie Burandt, Anna Szumelda 
2. Von Liebhaberei und wirtschaftlichem Nutzen - Wie in der Flusslandschaft Elbe Agrobiodiversität erhalten wird 71 Annemarie Burandt

3. Landwirtschaft und Lebenswirklichkeiten in kleinen landwirtschaftlichen Betrieben in Ost- und Südostpolen Beispiele aus den Landkreisen Lubartów und Krosno 93 Anna Szumelda

4. Die Natur des Ländlichen - Zur Konzeption gesellschaftlicher Natur- und Geschlechterverhältnisse in ländlichen Räumen..... 119 Tanja Mölders

5. Fazit zum Politikfeld Ländliche Entwicklung - Der Wandel ländlicher Räume als Wandel gesellschaftlicher Naturverhältnisse.

Tanja Mölders, Annemarie Burandt, Anna Szumelda

\section{Teil III: Agro-Gentechnik als Politiken der Naturgestaltung}

1. Einführung in das Politikfeld Agro-Gentechnik - Zwischen Bürokratisierung, Ökonomisierung von Governance und Politisierung 147

Beate Friedrich, Daniela Gottschlich, Jędrzej Sulmowski

2. Lokale und regionale Konflikte um Agro-Gentechnik Beate Friedrich

3. Die verschwiegenen politischen Momente in der AgroGentechnik-Debatte in Polen. Jędrzej Sulmowski 
4. Demokratisierung gesellschaftlicher Naturverhältnisse durch Widerstand und Gegenmacht - Die Bewegung gegen AgroGentechnik als Teil einer neuen Demokratiebewegung ............ 193 Daniela Gottschlich

5. Fazit zum Politikfeld Agro-Gentechnik - Umkämpfte Naturgestaltung, umkämpfte Politikgestaltung

Daniela Gottschlich, Jędrzej Sulmowski, Beate Friedrich

\section{Teil IV: Fazit und Reflexion}

1. Politiken der Naturgestaltung als Denk- und Handlungsräume für Anpassung, Kooperation, Widerstand und Alternativen ..... 227 Daniela Gottschlich, Tanja Mölders

2. PoNa als inter- und transdisziplinäres Experiment in der Sozial-ökologischen Forschung 243

Matthias Bergmann, Daniela Gottschlich, Tanja Mölders, Engelbert Schramm

Autor_innenverzeichnis 265

Literaturverzeichnis. 269 


\section{Abbildungs- und Tabellenverzeichnis}

\section{Abbildung 1, Kapitel 1.2.3:}

Entwurf eines kritisch-emanzipatorischen Nachhaltigkeitsverständnisses

\section{Abbildung 1, Kapitel II.2.2.3:}

Schutz und Nutzung von Agrobiodiversität als Ausdruck gesellschaftlicher Naturverhältnisse

Abbildung 1, Kapitel IV.2.3.2:

Transdisziplinärer Forschungsprozess (ISOE)

Tabelle 1, Kapitel II.3.1:

Polens Landwirtschaft in Zahlen, Daten des Agrarzensus 2010. 100

\section{Tabelle 1, Kapitel III.4.3:}

Formen gewaltfreier Aktion

\section{Tabelle 2, Kapitel III.4.3.1:}

Widerstand und Gegenmacht der Bewegung gegen Agro-

Gentechnik auf der ersten Eskalationsstufe. 205

\section{Tabelle 3, Kapitel III.4.3.2:}

Widerstand und Gegenmacht der Bewegung gegen Agro-

Gentechnik auf der zweiten Eskalationsstufe 208 
Tabelle 4, Kapitel III.4.3.3:

Widerstand und Gegenmacht der Bewegung gegen Agro-

Gentechnik auf der dritten Eskalationsstufe 214

Tabelle 1, Kapitel IV.2.3.1:

Typologie der Transdisziplinarität 255 


\section{Abkürzungsverzeichnis}

$\mathrm{AbL}$

ANT

AR

ARFE

AWU

BMBF

BMELV

BNatschG

BÖLW

BR

BRFE

BSE

BUKO

BUND

Bt-Toxin

CDU

CSU

DFG

DLR

DNR

DVS

EFSA

ELER

ESU

EU

FDP

FDP

FONA

GAP

Arbeitsgemeinschaft bäuerliche Landwirtschaft

Akteur-Netzwerk-Theorie

Arche-Region

Arche-Region Flusslandschaft Elbe

Annual Work Unit

Bundesministerium für Bildung und Forschung

Bundesministerium für Ernährung, Landwirtschaft und Ver-

braucherschutz

Gesetz über Naturschutz und Landschaftspflege/ Bundesnatur-

schutzgesetz

Bund Ökologische Lebensmittelwirtschaft

Biosphärenreservat

Biosphärenreservat Flusslandschaft Elbe

Bovine spongiforme Enzephalopathie, umgangssprachlich

„Rinderwahn“

Bundeskoordination Internationalismus

Bund für Umwelt und Naturschutz Deutschland

Bacillus thuringiensis-Toxin

Christlich Demokratische Union Deutschlands

Christlich-Soziale Union

Deutsche Forschungsgemeinschaft

Deutsches Zentrum für Luft- und Raumfahrt

Deutscher Naturschutzring

Deutsche Vernetzungsstelle Ländliche Räume

Europäische Behörde für Lebensmittelsicherheit

Europäischer Landwirtschaftsfonds für die Entwicklung des

ländlichen Raums

European Size Units

Europäische Union

Freie Demokratische Partei

Forum Debaty Publicznej (Forum Öffentlicher Debatte)

Forschung für Nachhaltigkeit

Gemeinsame Agrarpolitik 
GbR/ GbRs Gesellschaft/en bürgerlichen Rechts

GEH e. V. Gesellschaft zum Erhalt alter und gefährdeter Haustierrassen e. V.

GenTG Gentechnikgesetz

GG Grundgesetz

GID Gen-ethischer Informationsdienst

$\mathrm{GmbH} / \mathrm{GmbHs}$ Gemeinschaft/en mit beschränkter Haftung

$\mathrm{GMO} / \mathrm{GMOs}$ genetically modified Organism/s

GVO/GVOs gentechnisch veränderte/r Organismus/en

GV Pflanzen gentechnisch veränderte Pflanzen

ISOE Institut für sozial-ökologische Forschung

IP Interviewpartner_in

KMU/ KMUs kleine/s und mittlere/s Unternehmen

KSOW Krajowa Sieć Obszarów Wiejskich, Nationales Netz Ländlicher Räume in Polen

MON810 Gentechnisch veränderte Maissorte

MON steht für Monsanto, den Entwickler und Patentinhaber der Maissorte. 810 ist die firmeninterne Produktbezeichnung

NABU Naturschutzbund Deutschland

$\mathrm{NGO} / \mathrm{NGOs}$ Nichtregierungsorganisation/en

NNA

Niedersächsischen Naturschutzakademie

OECD

Organisation für wirtschaftliche Zusammenarbeit und

Entwicklung

PoNa Forschungsnachwuchsgruppe „Politiken der Naturgestaltung. Ländliche Entwicklung und Agro-Gentechnik zwischen Kritik und Vision"

RNE Rat für Nachhaltige Entwicklung

SÖF Sozial-ökologische Forschung

tdPrax Stärkung der transdisziplinären Forschungspraxis - Synopse und Anleitung für Konzepte, Methoden und Qualitätsmanagement

TTIP Transatlantic Trade and Investment Partnership

VDW Vereinigung Deutscher Wissenschaftler und Wissenschaftlerinnen

WTO World Trade Organization 